International Journal of Engineering \& Technology, $7(3.10)(2018) 73-76$
International Journal of Engineering \& Technology
WPC
Website: www.sciencepubco.com/index.php/IJET
Research paper

\title{
Auto Result Analyser and Sender
}

\author{
A.M.Afrin Zulaiha ${ }^{1}$, Dr. K.A. Mohamed Junaid ${ }^{2}$

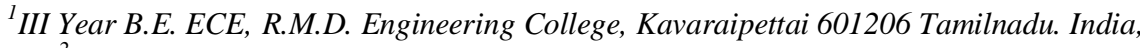 \\ ${ }^{2}$ Principal, R.M.K Engineering College, Kavaraipettai - 601206 Tamilnadu India \\ "Corresponding author E-mail: afrinzulaiha@gmail.com,
}

\begin{abstract}
Web development has taken various steps to strengthen knowledge base activities and supporting the client ideas. A pertinent portal is generated to maintainand store records which can be used by many educational institutions. The enhancement is made by using auto notification of electronic mail by specifying the email address for each student. Auto Result Analyser and Sender (ARAS) proves out to be more task effective and predominantly reliable. The information regarding student enrolment, internships are provided.The real-time reporting can be done once results are published and sent as mail/SMS to the registered students/parents. The performance wise comparison among various colleges and the students can be carried out with the usage of statistical analysis. SQL supports all the queries related to the database and the table insertions.
\end{abstract}

Keywords: Auto Result Analyser and Sender (ARAS), Electronic Mail (EM), Structured Query Language (SQL).

\section{Introduction}

In order to replace the current paper records and design a systematic format by which the students' academic progress as well as the automatic feature of result generation is done. ARAS is maintained through a secure, online interface embedded in the institution's website. The proposed system utilizes the necessary user authentication and sorting out only the relevant information for each individual. Additionally, it ensures security and reduces network traffic. The main feature includes enhancing client-server relationships, maintaining user loyalty, streamlining network communication and other endeavors. Electronic mail can be sent and accessed between various users of different computer systems running on heterogeneous operating systems. A network called JANET[2], which is the most effective part of the internet, which can be exchanged with most of the national and international networks. The mail delivered to the students gives the details of their registered subjects indicating the grades along with the credits of the respective subjects. This paper discusses how the standard results are analysed and the email lists with the generation of electronic mail to enhance the goals of the organization.

The student users' interface allows the students to access the information online. The processing time and the response generating time are greatly reduced to enhance the speed way of access. The student records are delivered to the users such that to ensure conformity of data[2]. The records and results of all the students are maintained with a simple interface.This paper mainly focuses on the facilities like online registration and profile creation of student with email generation of results in an intelligible manner.

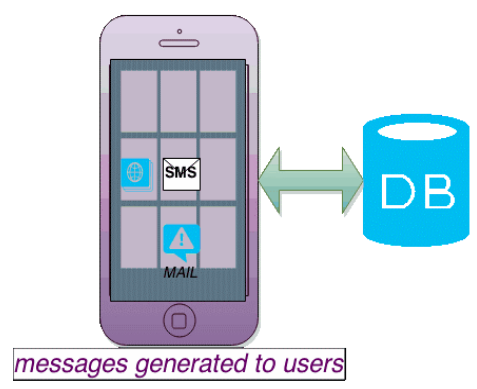

Figure 1

The use of in-trend technologies like mail/SMS is considered the added advantage of ARAS.

\subsection{Purpose}

The major purpose is to design and develop a web portal feature that contains the profile details of the student, information about the institution. The process is compatible and could easily extend support in all platforms. The performance of student and college wise comparison are tabulated with a graphical format. High overload could be easily brought under control.

\subsection{Objective}

The main objectives of ARAS are:

- Maintain, enhance and retrieve records

- Update result and notify it

- Enrollment and registration of student is done

- Performance of college and student is compared

- Ensure safe transfer of data and result

- Prevents site crash and unauthorized access 
- Proves out to be more task efficient

\subsection{Organization of Paper}

The paper is partitioned in and as follows:

Section II explains the methodology with the functional and non functional requirements. Section III provides the details of the various technologies used. Section IV covers the details of the system approach. Section V deals with the system benediction. Finally, section VI is the conclusion.

\section{Methodology}

A proper methodology starts with a good understanding of the necessary user requirementand system feature. A requirement is the description in order to fulfill the purpose. Every process model has certain perspective to be used. The basic requirements needed for the design of ARAS are:

- $\quad$ Every student is provided with their own identity

- An unique username is created for all staff member and student

- Enable to update his/her own personal details systematically

- Update about the college is also made

- The assessment details are periodically updated from staff side

- $\quad$ SMTP is used for secure email[4] to transfer POP3 is used to receive email

- Requirements are broadly categorized as functional and non functional

\subsection{Software Progression Model}

The software model is a method to create and enhanced system with the necessary series of steps as a lifecycle form. The parent model is broken down into a series of child models such as inputs and finished development as output form.

\section{$2.2 \quad$ Functional Requirement}

ARAS system aims to improve the efficiency and performance of the institution by managing and handling the information. The major functional requirements are the administrators and students. The admin has the power to update/enable/disable the users.

\subsection{Non Functional Requirement}

\subsubsection{Performance Requirement}

The response time is quick with the developed ARAS system. It can manage more load easily when query generation and processing are done concurrently.

\subsubsection{Safety Requirements}

The database backup[1]could be made to retrieve the lost database and crashing of database occurs due to operating system failure or any other malware.

\subsubsection{Recovery Requirements}

Backup generation could be utilized to fetch the data effectively.

\subsubsection{Security Requirements}

Admin decides the access rights for everyone. Password entry and authentication are done to ensure the secure data transfer.

\subsection{Database Delineation Process}

Database plays a more critical role in almost all areas on the prominent fields are business,law, engineering,medicine,etc.
The implicit properties of the database are:

- A database facilitates the process of defining, constructing and sharing among various users.

- Querying the database[7] to retrieve data and generate reports by application program

- Multiple users can share and access

\section{Technologies Used}

\subsection{PHP}

PHP is hypertext preprocessor, a powerful server-side scripting language which is used for developing dynamic web pages and also to make it interactive.

\subsection{SQL}

SQL stands for Structured Query Language which is to allow access to database and manipulate them.

\subsection{HTML}

HTML is a hypertext markup language which is in reality a backbone of designing any website.

\subsection{CSS}

Cascading Style Sheets (CSS) is a style sheet language used for describing the look and formatting of a document.

\section{Literature Survey}

The Electronic Mail (EM) which is also known as e-mail is the computer based approach of sending messages even if the user is not logged in [4].

Originally, email messages could handle only simple text but now complicated formats such as graphics and word processing documents are effectively managed [6].

The conventional system of approach involved the result announcement mainly done using mass media open to all people. Previously, most of the institutions had heavy paper/records which proved to be the drawback[2].

At first, the information is conveyed to the student and the website of the respective institution provides the link which carries to another HTML page and the processing takes more time[2]. It could even result in network traffic and lead to server crash. Hence, it becomes a tedious process.

Later it was diverted into websites and public portals[1] thereby it was accessed by the student with the details of roll number. Further it was improved in which mobile phones were utilised to handle the SMS request and send back the result[4].

The major disadvantages are the server load, less response time, performance delay, network traffic, unexpected server crash. Now in the proposed system we have planned in such a way that results are notified to the students' registered mail ids/mobile. It further ensures that there are no unwanted ads, popup and third party sites disturbances focussing mainly on clarity and security of results.

\section{System Approach}

\subsection{Admin Workflow Analysis}

ARAS is a web portal which is under the overall control of an admin. Admin can add his subordinates as users in the system with individual usernames and passwords. All the addition/deletion/updation works[5] can be done. Admin is authoritative for publishing the result. The number of servers to be used has to be decided by the admin . 


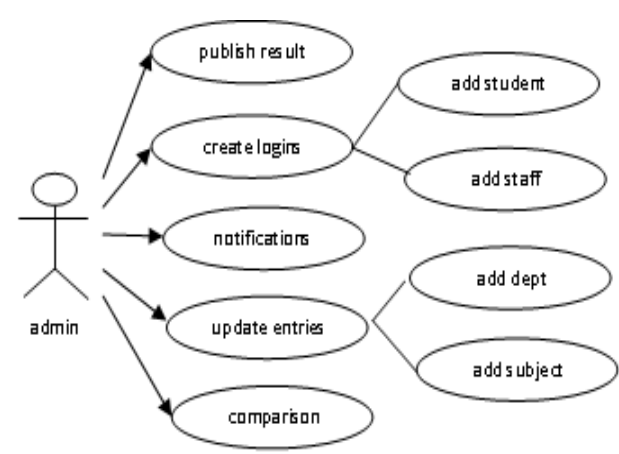

Figure 2

\subsection{User Restraints}

ARAS provides the users with the feature to add/update/delete student or teacher records providing database access. The user is allocated the job of collecting all student related information and adding them in the database. ARAS maintains the database for the complete course till all the semesters are completed to retrieve and display the details of grades/performance[6]. The student/institution can access the performance details.

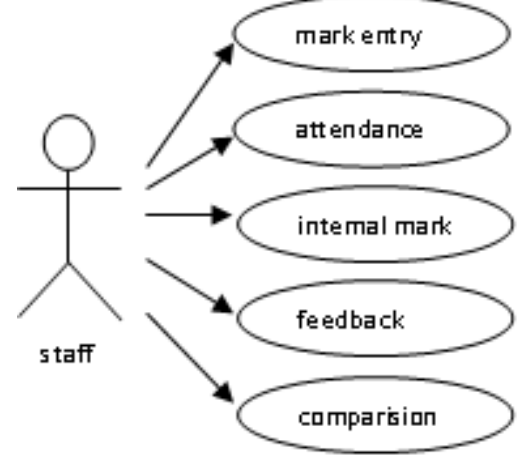

Figure 3
The data entry work for the result feeding process is the restraint of the user which is to be done in a short period of time after approved by the chief examiner. The feeding will be done through an interface linked to the main database. ARAS includes the internal assessment details during feeding process and the values are stored in database records[2].

\subsection{Internal Assessment Process}

The internal assessment process is carried out in almost all the universities these days which includes the percentage of marks from the internal exams and the attendance of the students. Though separate strategies are adopted, a common system of assessment is one which involves adding the total assessment to the marks obtained in the external exams. The internal marks for the students have to be fed to ARAS by the college authorities.

\subsection{Result Notification Process}

The result publication is annexed with the notification of the same which is started in advance for the sufficient time to initiate and send the notifications. The time head-start for notification before actual publication depends on the number of mails/messages to be sent and the number of available servers . The technical person makes sure that the notification is completed fully before the result is published on the web portal.

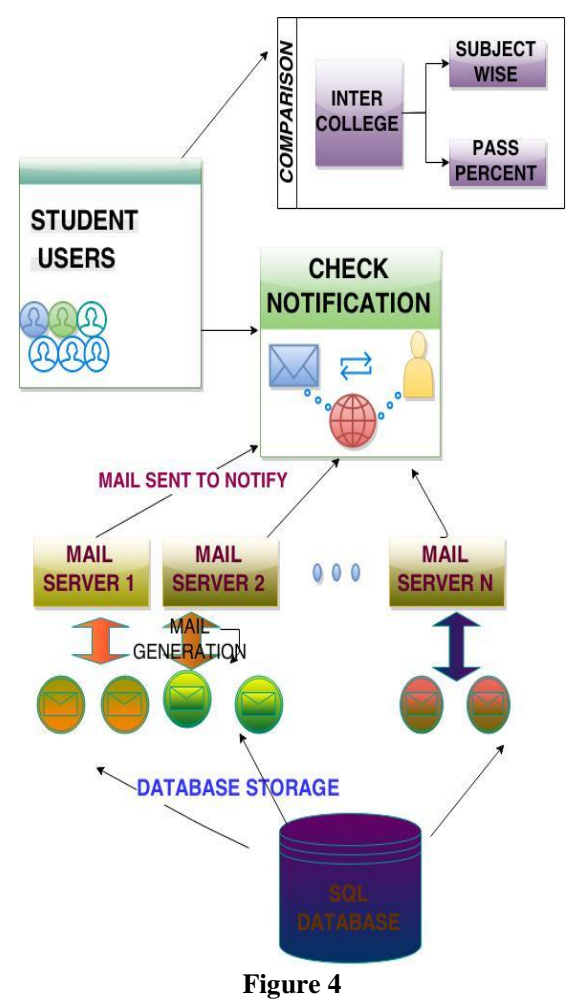

\subsection{Result Analysis Strategy}

The result analysis part is an important feature of ARAS which is done by comparing the grades of students as per the following criteria:

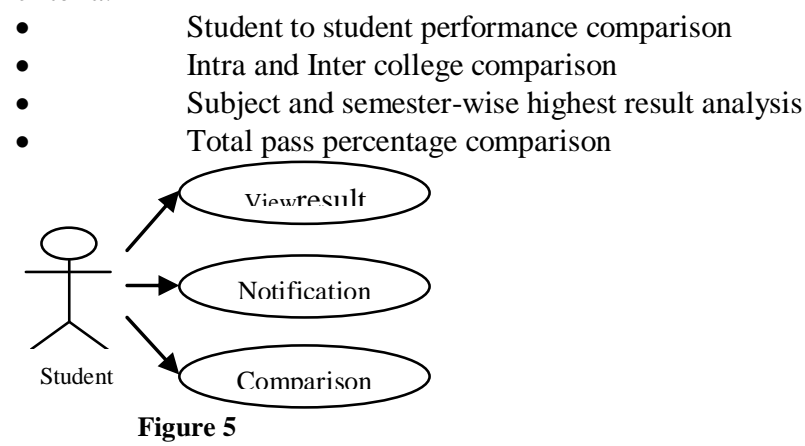

\section{Benediction}

\subsection{Usability:}

Usability denotes the ease of use and learnability. It generally includes studies of clarity of websites and computer programs.Hence the ARAS system is said to have the high level of usability which is implemented in PHP because of its popularity, independency, security and wide usage range.

\subsection{Reliability:}

Reliability is an attribute that consistently performs according to its specification. ARAS system is reliable to a high extend that every student will get his/her result a few moments after publishing. Correctness of result tends to score cent as the result is obtained from direct source of authority. Regression based data mining algorithm is used for the comparison of data[5].

\subsection{Security:}

The important parameter that every system should possess is the security. Security prevents/protects the system from unauthorized access. Security measures also barricades the intruders into the 
system. Any user can log in into the system only if they are validated. Validation is done through means of username and password[6]. Student results are secured through the standards and mailing agents such as Gmail, yahoo etc.Pretty good privacy(PGB) is the most standard protocol which is to encrypt and decrypt the texts, files, directories etc.PGP evolves more features that support to create the encrypted messages. It ensures confidentiality and handles multiple messages[4].Integrity checking can be perfectly done with PGP. The public key encryption and symmetric key encryption are being combined for safety transfer of messages. When the symmetric key is utilized only one time it is known as session key. If the delivery message is altered then PGP considers integrity property.

\subsection{Maintainability:}

In general terms of Engineering, maintainability is the ease with which a product can be maintained in order to isolate from defects or the roots of defects, correcting and repairing the faulty parts. The two main components are serviceability and repairability. Serviceability denotes the ease of conducting scheduled inspection. Repairability is the ease of restoring service after failure.

\section{Conclusion}

The paper assists in generating email automatically to all the registered students. Safe storage of data is possible in the repository. The delay in processing gets reduced. The stakeholders can get the required data easily. All possible ways of comparison are done statistically.

\section{References}

[1]. Zhibing Liu, Huixia Wang,Hui Zan "Design and implementation of student information management system." 2010 International symposium on intelligence information processing and trusted computing. 978-0-7695-4196-9/10 IEEE.

[2]. S.R.Bharamagoudar, Geeta R.B., S.G.Totad "Web Based Student Information Management" 2013 International Journal of Advanced Research in Computer and Communication Engineering Vol. 2, Issue 6

[3]. M.A. Norasiah and A. Norhayati. "Intelligent student information system". 4th International conference on telecommunication technology proceedings, Shah Alam, Malaysia, 0-7803-7773-7/03 2003 IEEE.

[4]. O. Awodele, E. R. Adagunodo, A. T. Akinwale, S. Idowu and M Agbaje "An Improved SMS User InterfaceResult Checking System " 2009 Interdisciplinary Journal of Information, Knowledge, and Management Volume 4

[5]. Jin Mei-shan1 Qiu Chang-li 2 Li Jing 3. "The Designment of student information management system based on B/S architecture". 978-1-4577-1415-3/12 2012 IEEE.

[6]. He, Z. and J. Zheng. Design and Implementation of Student Attendance Management System Based on MVC in Management and Service Science, 2009. MASS '09. International Conference on. 2009.

[7]. Ibrahim A.S.Muhamadi, M.A Zaidan, A.A Zaidan, B.B Zaidan, "Student Record Retrieval System Using knowledge Sharing", International Journal of Computer Science and Network Security (IJCSNS), Vol.9, No.6 , ISSN : 1738-7906, pp. 97-106, 30 June (2009), Seoul, Korea. 